
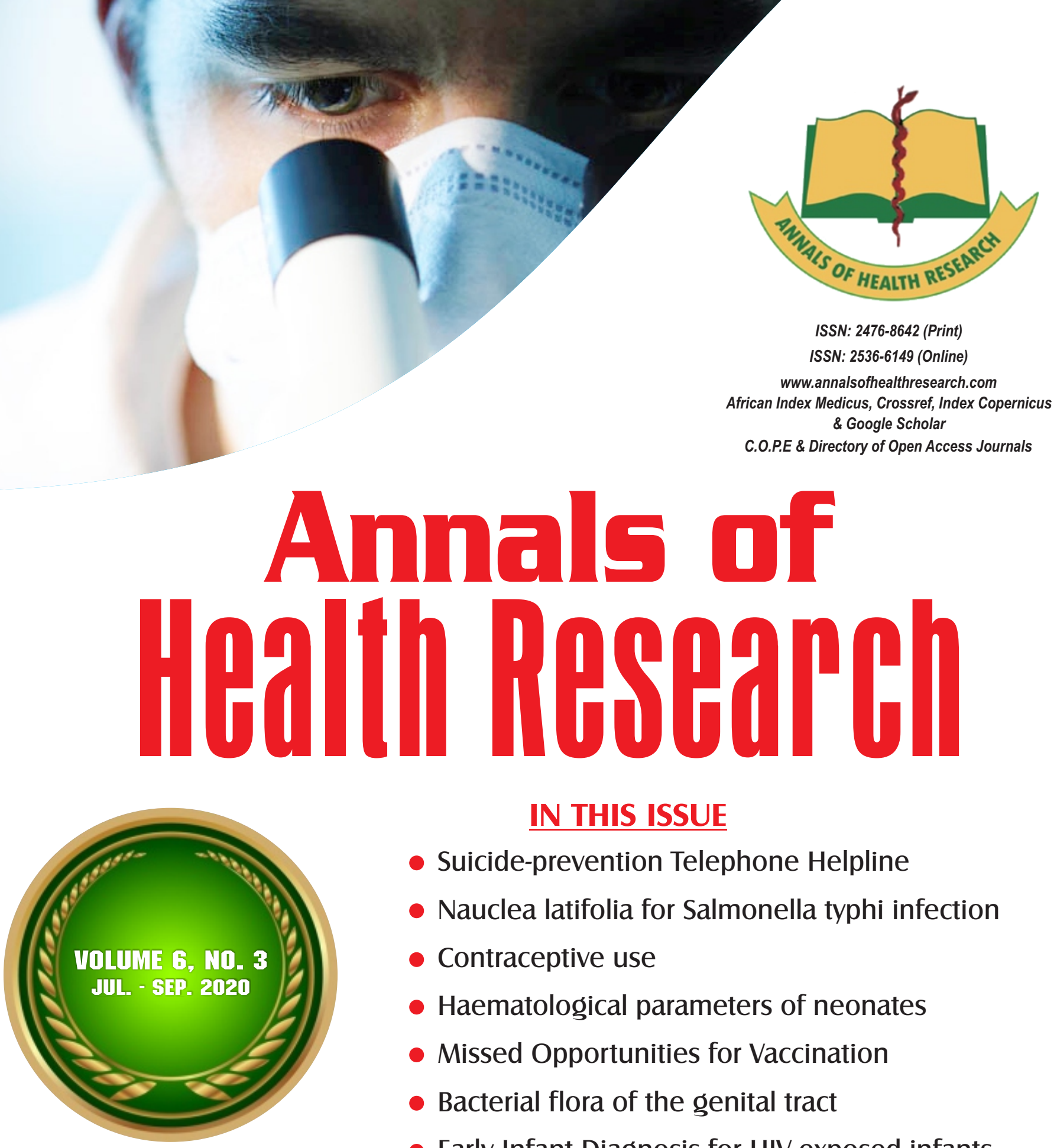

IN THIS ISSUE

- Suicide-prevention Telephone Helpline

- Nauclea latifolia for Salmonella typhi infection

- Contraceptive use

- Haematological parameters of neonates

- Missed Opportunities for Vaccination

- Bacterial flora of the genital tract

- Early Infant Diagnosis for HIV-exposed infants

- Bone markers and cardiovascular risk factors

- Attitude to termination of pregnancies

- Herpes zoster ophthalmicus

- Neonatal hyperinsulinaemic hypoglycaemia

- Paediatric perineal injury

PUBLISHED BY THE MEDICAL

AND DENTAL CONSULTANTS ASSOCIATION

OF NIGERIA, OOUTH, SAGAMU, NIGERIA.

www.mdcan.oouth.org.ng 


\title{
Characteristics of HIV/ Malaria co-infected mothers who did not access early infant diagnosis for their HIV-exposed infants in Benin-City, Nigeria Sadoh AE*, Eki-Udoko FE

\author{
Department of Child Health, University of Benin Teaching Hospital, Benin-City, Nigeria
}

"Correspondence: Prof. AE Sadoh, Department of Child Health, University of Benin-City, Nigeria. E-mail: evawere.sadoh@uniben.edu; ORCID - https://orcid.org/0000-0002-5681-9547.

\begin{abstract}
Background: Perinatally-acquired HIV infections rapidly lead to death within one to two years of life. Early infant diagnosis (EID) is the key to early identification with the prompt commencement of treatment. This has been shown to reduce morbidity and mortality. In Nigeria, only $21 \%$ of HIV-infected infants are on ARTs.

Objective: To determine the characteristics of HIV/Malaria co-infected mothers who did not access EID for their HIV- exposed infants.

Methods: HIV infected mothers were recruited from three hospitals during the last trimester of pregnancy and were tested for peripheral and placental malaria at delivery. Socio-demographic and some clinical characteristics were obtained using a proforma. Following delivery, the babies were commenced on the Prevention of Mother to Child Transmission (PMTCT) protocol for HIV including prophylactic Nevirapine therapy, infant feeding counselling and performance of DNA PCR at 6-8 weeks of life. Mothers whose infants did not have DNA PCR results were categorized as having not accessed EID services.

Results: Of the 162 infants, only $101(62.4 \%)$ had a DNA PCR test done. The mean age of mothers who did not access EID (31.84 \pm 4.8 years) was significantly higher than for mothers who accessed EID ( $30.58 \pm 3.09$ years) $(\mathrm{p}<0.0001)$. Mothers with low levels of education $(p=0.006)$, cohabiting mothers $(p=0.0067)$ and HIV serodiscordant couples $(p$ $=0.0029$ ) were more likely not to access EID.

Conclusion: Education, marital status and HIV serodiscordance are significant determinants of accessing EID services. Assessment of these factors at first contact with a pregnant HIV-infected woman allows risk categorisation for default from EID.
\end{abstract}

Keywords: Early infant diagnosis, HIV-exposed infants, HIV-positive mothers, Malaria, PMTCT.

\section{Introduction}

Human Immunodeficiency Virus (HIV) infection is a major challenge in sub-Saharan Africa, being a major contributor to morbidity and mortality in children. ${ }^{[1]}$ An estimated 120,000 of the global one million deaths from HIV/AIDS in 2016 occurred amongst children aged less than 15 years. Sub-Saharan Africa accounts for $64 \%$ of new HIV infections globally. [2] A majority of HIV infections in children in these settings are perinatally-acquired and it is well documented 
that such infections rapidly lead to mortality if untreated with up to $50 \%$ of infected infants dying before the age of two years. ${ }^{[3,4]}$ It has also been documented that early initiation of antiretroviral therapy reduces the morbidity and mortality in these children. [5] Therefore, it is imperative that perinatal transmission of HIV infection is not only prevented but that early detection should be ensured, to enable the appropriate and timely introduction of highly active antiretroviral therapy (HAART).

The prevention of mother-to-child transmission (PMTCT) of HIV is a comprehensive programme which includes primary prevention of HIV among women of childbearing age, preventing unwanted pregnancy among women living with HIV, preventing transmission from a woman living with HIV to her infant and providing appropriate treatment, care and support to mothers living with HIV, their children and families. [6] The steps in the PMTCT cascade includes testing to identify HIV-infected women, the commencement of antiretroviral treatment (ART) for the prevention of maternal transmission, testing of the HIV-exposed infant (early infant diagnosis) and initiation of ARTs in the HIV infected child. [7]

Early Infant Diagnosis (EID) as one of the components of the PMTCT strategies, entails testing of all HIV-exposed babies to determine their HIV infection status by detecting the presence of HIV Deoxyribose Nucleic Acid (DNA) using Polymerase Chain Reaction (PCR) tests. ${ }^{[7,8]}$ This virological testing which should be carried out between the sixth and eighth week of life, is a major challenge in resourcelimited settings. [9] The cost of virological tests, non-availability and lack of access to the test have been identified as barriers to effective EID programmes. [9,10] The more readily available antibody-based tests are not suitable for children aged below 18 months of age as maternally- transferred antibodies may persist until 18 months and may give false-positive results.

EID confers substantial benefits to both the HIVinfected and uninfected infant, their families and programmes providing PMTCT services. ${ }^{[10]}$ For the infected child, it enables early initiation of ARTs which has been shown to reduce morbidity and mortality. Infant feeding options are also facilitated by knowledge of the child's status preventing situations of mixed feeding while encouraging exclusive breastfeeding or replacement feeding for the HIV-infected child. A negative DNA PCR test is reassuring to the family of the child and this enables bonding when such babies have to be raised by extended family in the event of maternal death or disabilities. Early discontinuation of prophylactic ART is facilitated by a negative DNA PCR test result and thus prevents the occurrence of drug resistance. EID results are useful in assessing the effectiveness of PMTCT programmes and a high proportion of negative results improves the morale of health care workers in the PMTCT programmes.

Ciaranello et al [10] in a review, had highlighted the loss to follow-up at key steps of the EID cascade of care, which include infant presentation to care, test offer by a health care worker, test acceptance by caregiver/parents, specimen collection and processing, the return of result to the health care worker and parents/caregivers and linkage to HIV care. The impact of such loss to follow-up may include poor outcomes, increased cost and poor costeffectiveness of EID programmes. ${ }^{5,10]}$

Global reports indicate that $82 \%$ of pregnant women were on ARTs for the prevention of mother-to-child transmission which was equal to the global average in 2017. [11] A systematic review on the accessibility of EID services in sub-Saharan Africa between 2004 and 2014, found that only $3-58 \%$ of children received a 
virological test within the first two months of life falling short of the global target of $80 \%$. [9] Nigeria has a high rate of new HIV infections in children with 37,000 new infections recorded in 2016. [2] Although the PMTCT coverage is improving in Nigeria, coverage was $30.2 \%$ in 2015 , lower than the $80 \%$ target and also lower than the African average of $82 \%$. [2, 11] Nigeria also has low rates of EID and the proportion of HIV-infected children on ART (21\%). [2] If caregivers do not access EID services for their HIV-exposed infants, the objective of identifying infected infants early and treating them will not be achieved. Many studies have highlighted the low rates of EID in Nigeria but only a few have characterized the mothers of these children who do not access EID. [12-17] To improve the uptake of EID services, it is important to identify those who do not access this service to enable targeted interventions. The twin scourge of HIV and malaria in pregnant women may portend negative outcomes for the infant in terms of transmission of both infections making it imperative for EID. [18] This study sought to describe the socio-demographic characteristics of a cohort of HIV/Malaria co-infected mothers who did not access EID for their HIV-exposed infants.

\section{Methods}

This comparative cross-sectional study was part of a larger study on congenital malaria among infants of mothers who are co-infected with malaria and HIV. The study was carried out at the three major public hospitals [University of Benin Teaching Hospital (UBTH), Central Hospital $(\mathrm{CH})$ and Stella Obasanjo Women and Children's hospital (SOWC)] that offer care (including ART) and PMTCT services to HIVinfected persons including pregnant women, children, adolescents and adults in Benin City.
The methodology has previously been reported. [19] The calculation of the minimal sample size using the standard formula [20], and based on a previously recorded prevalence, [21] yielded 162. Therefore, 162 newborns of mothers co-infected with HIV and malaria were recruited as subjects and 162 newborns of HIV-negative mothers with malaria were recruited as controls. The number of mothers from each of the study sites was assigned in a ratio of 4.5:3.8:1.7, proportionate to the number of HIV positive mothers who were delivered in each site the previous year. Thus 73, 61 and 28 mother-baby pairs were recruited from $\mathrm{CH}, \mathrm{UBTH}$ and SOWC respectively. The mothers were recruited from the antenatal clinics of the hospitals in the third trimester of their pregnancies after obtaining consent. Recruitment was done over six months from June to November 2013.

Socio-demographic details were obtained and documented for each mother. Mothers who had diabetes mellitus, sickle cell anaemia, who were on therapy for cancer and those on prophylactic or therapeutic cotrimoxazole were excluded as these could potentially affect the outcome variables of congenital malaria. At delivery, consecutive mother-infant pairs were enrolled in the study. Peripheral blood of mothers was tested for malaria parasitaemia while placental malaria was detected using histology. HIV testing (using the national algorithm), [22] and counselling is routinely offered to every mother during antenatal care visits. HAART is offered to HIV-positive mothers and their babies are commenced on the PMTCT protocol which includes nevirapine therapy and infant feeding counselling. The babies were referred to the UBTH HIV Clinic for DNA PCR testing at 6-8 weeks of life. The DNA PCR results were subsequently retrieved from the laboratory within six months of completion of recruitment. Since all the tests were carried out in the UBTH laboratory, any child for whom a result was not found was deemed not to have accessed the EID 
service. The subjects of this study were mothers who met the inclusion criteria of being HIVinfected, had peripheral malaria parasitaemia and or placental malaria as documented by histology.

\section{Ethical considerations}

Ethical approval was obtained from the Ethics and Research Committee of the University of Benin Teaching Hospital, Benin-City.

\section{Data analysis}

Data were entered into the Statistical Package for Social Sciences version 20 spreadsheet for statistical analysis. Mothers whose babies did not have DNA PCR results were categorized as haven not accessed EID services while those whose babies had results were categorized as haven accessed EID services. Continuous variables were summarized using means and standard deviation. The difference between means was tested using Student's t-test, while the comparison of proportions was done using the Chi-Squared test and Fishers Exact test as appropriate. The level of statistical significance was set at $\mathrm{p}$ values less than 0.05 at $95 \%$ confidence interval.

\section{Results}

The DNA PCR results were available for 101 $(62.4 \%)$ of the 162 infants delivered to mothers who were co-infected with malaria and HIV. The mean age of the mothers who accessed EID was $30.5 \pm 3.0$ years which was statistically significantly lower than $33.4 \pm 4.8$ years for mothers who did not access EID $(p<0.0001)$ (Table I). The mean age of the fathers of infants with DNA PCR results was $35.0 \pm 3.4$ years. This was also statistically significantly lower than $39.2 \pm 5.7$ years for fathers of infants without DNA PCR results $(\mathrm{p}<0.0001)$.
A majority of the mothers of the infants studied were married. There was a statistically significantly higher proportion of co-habitation (18; 29.5\%) among mothers of infants who did not access EID ( $p=0.0067)$. Parental education was statistically significantly associated with accessing EID. Of the 61 infants who did not access EID, $41(67.2 \%)$ of the mothers had primary education or lower compared to 36 (35.7\%) mothers of the 101 infants who accessed EID ( $p=0.0006)$. Similarly only $9(14.8 \%)$ of the fathers of infants who did not access EID had tertiary education compared to $36(35.6 \%)$ of fathers of children who accessed EID ( $p=$ $0.0154)$.

There was a significantly higher proportion of seropositivity among the fathers of infants who accessed EID (26; 25.7\%) compared to $4(6.6 \%)$ of those who did not access EID ( $p=0.0029)$. Of the 162 spouses/partners, $30 \quad(18.5 \%)$ were seropositive for HIV. Of these, $26(86.7 \%)$ of their infants accessed EID whereas only 75 $(56.8 \%)$ of 132 infants whose fathers were seronegative for HIV accessed EID.

Of the 63 mothers with parity of one, $53(84.1 \%)$ accessed EID compared to $20(37.7 \%)$ of those with a parity of 3 or more $(\mathrm{p}<0.0001)$. A majority $(85 ; 80.2 \%)$ of the mothers who lived in urban areas significantly accessed EID compared to those who lived in rural areas $(p<0.0001)$.

\section{Discussion}

In this study, only $62.4 \%$ of the infants had DNA PCR results, which is much lower than the target of $80 \%$ of HIV-exposed infants for access to EID, as stipulated by the National Strategic Plan for 2010-2015. [23] This figure is comparable to $68.3 \%$ reported by Oladokun et al. [17] in 2010 and $71 \%$ in an intervention programme in southeastern Nigeria. [24] This suggests that a large proportion of HIV-exposed infants do not get 
the opportunity to be tested and their HIV status may not be known. Failing to access EID means that those who are seropositive for HIV may not commence HAART promptly thus, creating the risk of suffering morbidities and even death before their status is detected.

Table I: Socio-demographic characteristics of mothers who accessed and mothers who did not access early infant diagnosis for their HIV-Exposed infants

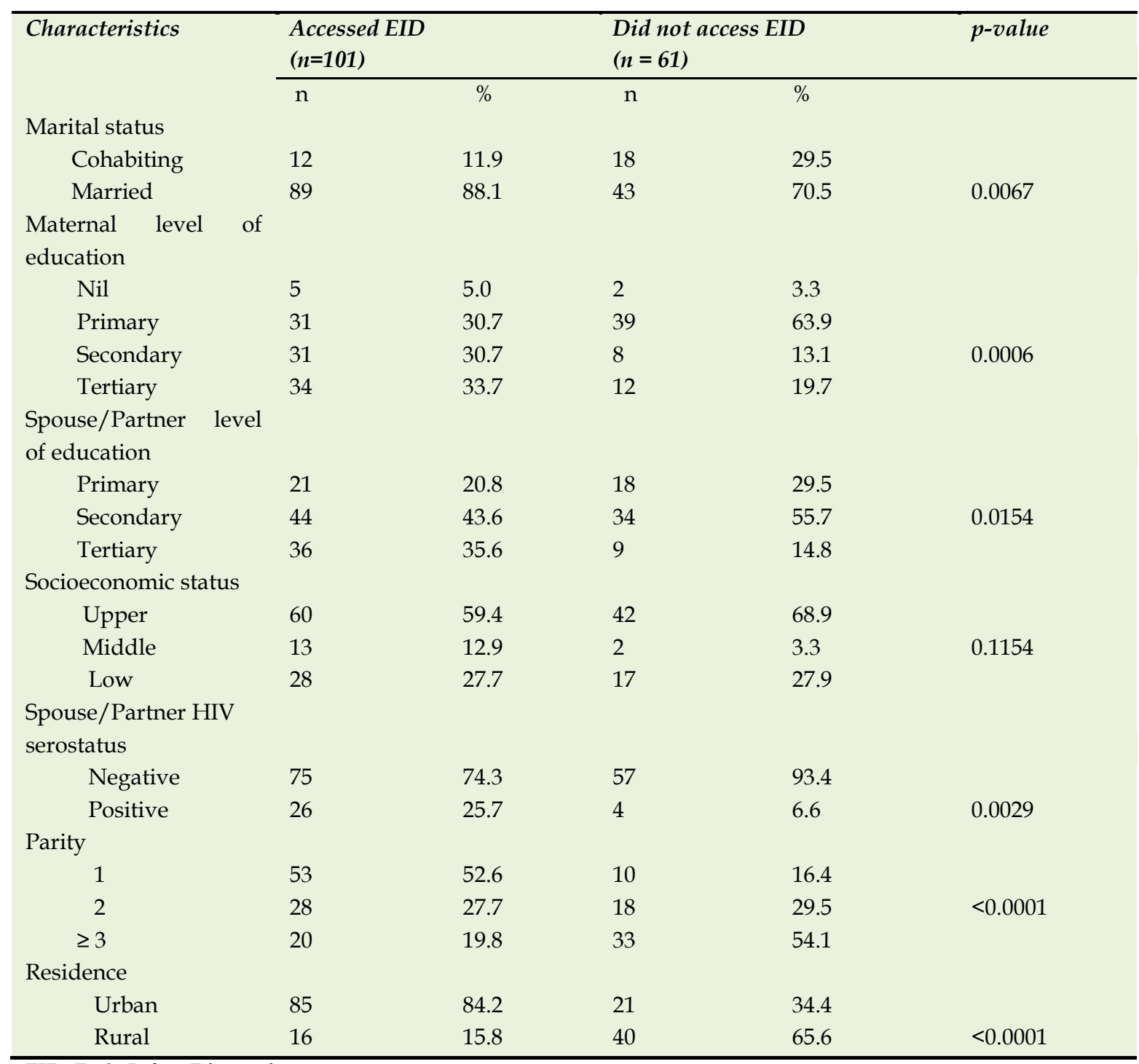

EID- Early Infant Diagnosis

In a review of EID in low-resource settings, it was noted that many children do not enter the EID cascade, especially those whose maternal HIV serostatus is unknown. [10] In the review, it was observed that most EID programs target infants of mothers whose HIV serostatus is already known and this strategy may miss testing for more than half of truly HIV-exposed infants. ${ }^{[10]}$ While this may be true, it is pertinent to also note that even amongst those with known HIV serostatus, there is a significant proportion of drop-out similar to the finding in the present study where almost $40 \%$ of HIVexposed infants did not access EID. Therefore, it may be instructive to know the characteristics of 
the mothers as this would enable targeted interventions to improve EID uptake.

The mothers of infants who did not access EID were older than those whose infants accessed EID. Their spouses/partners were also older than the spouses of mothers whose infants accessed EID. This may be as a result of the fact that a lot of the health education concerning HIV was targeted at young persons who are sexually active and so they are probably more knowledgeable concerning HIV and its prevention. This is further corroborated by the finding in this study that the mothers who accessed EID were better educated than those who did not. Similarly, the fathers of infants who accessed EID were also better educated. Higher levels of education have been associated with better uptake of EID in other studies. [17]

Also of note is the finding that mothers with higher parity were less likely to access EID, probably because they have had other children with no health problems and may be in denial of their HIV serostatus or of the possibility that HIV could be transmitted to their infants. It is also possible that HIV infection was acquired in the context of a long term relationship suggesting the possibility of infidelity, which may then prevent disclosure especially if there is serodiscordance. Non-disclosure has been identified as a potential reason for low uptake of PMTCT services by mothers who know their HIV status. [25] The association of multiparity with low uptake of EID in the present study is in keeping with the findings in a study that compared EID defaulters with those who did not access EID at all. [26] In that study, multiparity was associated with EID defaulters. Unfortunately, the authors of that work did not compare the EID defaulters with EID compliant mothers.

Family support has been associated with better outcomes in HIV care. ${ }^{[17,27]}$ In this study, it was noted that parents who were cohabiting were significantly less likely to access EID than those who were married. Infants from relationships in which both parents were seropositive for HIV were also more likely to access EID than infants of HIV serodiscordant parents. It is possible that parents who are HIV serodiscordant may not have disclosed their HIV serostatus, thus, making it impossible to benefit from partner and family supports. Partner support is a known important factor in HIV care. [27]

Previous studies have also suggested that distance may be a factor in the inability of mothers to access HIV care for themselves and their children. [26,28] This may have been a problem in the study population considering that DNA PCR testing was only available in one of the three study sites. The role of distance as a possible challenge may be corroborated by the finding that those who resided in rural areas were more likely not to access EID whereas all the study sites are located in urban areas. Collecting Dried Blood Spot (DBS) samples in the other hospitals could have mitigated the problem of transport as the infants would not be required to be physically present at the centre where the test is being done. This is now the standard of care in the study hospitals.

Socioeconomic status was not a significant determinant of EID uptake in the present study suggesting that education rather than finance is more important with regards to accessing EID care, especially since the testing is free. Although this study was carried out amongst HIV/ Malaria co-infected mothers, it is perceived unlikely that the mothers would be significantly different from other HIV-infected mothers who are free of malaria. The findings in this study may, therefore, be generalizable to HIV-positive mothers in the study locale. This study is limited by the fact that mothers and infants were not tracked to find out the reasons for the default and to identify the point in the EID cascade where they defaulted. 


\section{Conclusion}

Older age (for both mothers and their partners), multiparity, low level of education (for mothers and partners), cohabitating couples and HIV serodiscordance are the factors associated with not accessing EID. It is unacceptable for motherinfant pairs who have been in contact with the health care system to miss out on such lifesaving interventions. A tracking system should be designed to enable HIV-exposed infants to be sought out, tested and enrolled into care as indicated.

It is recommended that female education be encouraged and HIV serodiscordant couples should be supported in caring for their offspring as well as in maintaining a healthy relationship.

Authors' Contribution: SAE conceptualized the study, analysed and interpreted the data, wrote the initial draft of the manuscript. FEE contributed to the concept, data collection, analysis and interpretation. Both authors approved the final draft of the manuscript.

Conflict of interest: None declared.

Funding: Self-funded.

Publication History: Submitted 08 April 2020; Accepted 30 June 2020.

\section{References}

1. Arunda MO, Choudhry P, Ekman B, Asamoah BO. Under-five mortality and maternal HIV status in Tanzania: analysis of trends between 2003 and 2012 among AIDs indicator survey data. Global Health Action 2016; 9. doi:10.3402/gha.v9.31676.

2. Joint United Nations Programme on HIV/AIDS. UNAIDS DATA 2017. UNAIDS. Available at www.unaids.org/sites/default/files/media _asset/20170720_Data_Book_2017_en.pdf
3. National Agency for the control of AIDS. Fact sheet: Prevention of mother to child transmission (PMTCT), 2016. NACA, Nigeria 2016. Available at https://naca.gov.ng/factsheet-prevention-mother-child-transmissionpmtct-2016. Accessed 28th March 2018.

4. Newell M, Coovadia $\mathrm{H}$, Cortina -Borja $\mathrm{M}$, Rollins N, Gaillard P, Dabis F for Ghent International AIDS Society (IAS) Working Group on HIV infection in women and children. Mortality of infected and uninfected infants born to HIV-infected mothers In Africa: a pooled analysis. Lancet 2004; 364: 1236-1245.

5. Cotton MF, Rabie H. Impact of earlier combination antiretroviral therapy on outcomes in children. Curr Open HIV AIDS 2015: 10 : 12-17. doi: 10.1097/0000000000000117.

6. African Network for the Care of Children Affected by HIV/AIDS (ANECCA). Handbook of Paediatric AIDS in Africa. Tindyebwa D, Kayita J, Musoke P, Eley B, Nduati R, Tumwesigye N, Mwansambo C, Mbori-Ngacha D and Kieffer M (Eds). $4^{\text {th }}$ edition, USAID. 2017. Available at www.anecca.org.

7. Wettstein C, Mugglin C, Egger M, Blasere N, Salazar L, Estill J, et al. Missed opportunities to prevent mother-to-child-transmission in sub-Saharan Africa: systematic review and meta-analysis. AIDS 2012; 26: 2361-2373.

8. National AIDS and STIs control programme. National guidelines for HIV prevention, treatment and care. Federal Ministry of Health, Abuja, Nigeria 2016.

9. Bwana VM, Frompong C, Simulundu E, Mfinanga SG, Mboera LEG, Michelo C. Accessibility of services for early infant diagnosis of Human Immunodeficiency virus in sub-Saharan Africa: a systematic review. Tan J Health Res 2016; 18: 1-18. http/ /dx.doi.org/10.4314/hrb18i3.9. 
10. Ciaranello AL, Park J, Ramirex-Avila L, Treedberg KA, Walensky RP, Leroy V. Early infant HIV 1 diagnosis programs in resourcelimited settings: opportunities for improved outcomes and cost-effective interventions BMC Med 2011; 9: 59 Available at http:/ / www.biomedcentral.com/17417015/9/59. Accessed 23 November 2017.

11. World Bank. Antiretroviral therapy coverage for PMTCT (\% of pregnant women living with HIV. Available at https://data.worldbank.org/indication/SH. HIV.PMTCT.ZS. Accessed 12 ${ }^{\text {th }}$ June 2020

12. Iloh KK, Iloh ON, Ikefuna AN, Ibeziako NS, Ubesie AC Emodi IJ. Determinants of mother -to- child transmission of HIV despite PMTCT interventions in Enugu, Nigeria. South Afr J Child Health 2015; 9: 49-52.

13. Sadoh WE, Omoigberale AI, Esene HA, Onakewhor JUE. Mother-to-child transmission of HIV at the University of Benin Teaching Hospital, Benin City, Nigeria. Sahel Med J 2008; 11: 118-124.

14. Anoje C, Aiyenigba B, Suzuki C, Badru T, Akpoigbe K, Odo M, et al. Reducing motherto-child transmission of HIV: findings from an early infant diagnosis program in the south-south region of Nigeria. BMC Pub Health 2012; 12: 184.

15. Oyesakin AB, Akinsulie A, Oniyangi $\mathrm{O}$, Audu LI, Ogunfowokan O. PMTCT programme reduce vertical transmission of HIV in Abuja, Nigeria. Niger J Paediatr 2016; 43: 166-169.

16. Ugochukwu EF, Kalu SO. Early infant diagnosis of HIV infection in southeastern Nigeria: prevalence of HIV infection among HIV exposed babies. West Afr J Med 2009; 29: 3-7.

17. Oladokun RE, Brown B, Osinusi K. Loss to follow-up rates, reasons and associated risk factors among mother-infant pairs in a prevention of mother-to-child transmission programme (PMTCT) in Nigeria: a casecontrol study. Niger J Paediatr 2006; 33: 7984.

18. King CC, Ellington SR, Kourtis AP. The role of co-infections in mother-to-child transmission of HIV. Curr HIV Res 2013; 11: 10-23.

19. Eki-Udoko FE, Sadoh AE, Ibadin MO, Omoigberale AI. Prevalence of congenital malaria in newborns of mothers co-infected with HIV and malaria in Benin City. Infect Dis 2017; 49: 609-616. doi:10.1080/23744235.2017.1312667

20. Araoye MO. Research Methodology with Statistics for Health and Social Sciences. Nathadex Publishers, Ilorin 2003.

21. Perrault SD, Hajek J, Zhong K, Owino SO, Sichangi M, Smith $\mathrm{G}$, et al. Human immunodeficiency virus co-infection increases placental parasite density and transplacental malaria transmission in Western Kenya. Am J Trop Med Hyg 2009; 80:119-125.

22. Federal Ministry of Health, Nigeria. National Guidelines for Paediatric HIV and AIDS Treatment and Care 2010; 978:48611-48626.

23. National Agency for the Control of AIDS. National HIV/AIDS strategic plan 2010-2015 Available at http://www.ilo.org/wcmsp5/groups/publi c/---ed_protect/---protrav/--ilo_aids/documents/legaldocument/wcms_ 146389.pdf. Accessed 25 June 2018.

24. Pharr JR, Obiefune MC, Ezeanolue CO, Osuji A, Ogidi AG, Gbadamosi S, et al. Linkage to care, early infant diagnosis and perinatal transmission among infants born to HIV infected Nigerian mothers: evidence from the healthy beginning initiative. J Acquir Immune Defic Syndr 2016; 72: S156-S160. 
25. Spangler SA, Onono M, Bukusi EA, Cohen CR, Turan JM. HIV-positive status disclosure and use of essential PMTCR and maternal health services in rural Kenya. J Acquir Immune Defic Syndr 2014; 67: S236-S242.

26. Anigilaje EA, Ageda BR, Nweke NO. Barriers to uptake of prevention of motherto -child transmission of HIV services among mothers of vertically infected HIV seropositive infants in Makurdi, Nigeria. Patient Preference Adherence 2016; 10-57-72.
27. Iwelunmor J, Ezeanolue EE, Airhihenbuwa C, Obiefune MC, Ezeanolue CO, Ogedengbe GG. Socio-cultural factors influencing the prevention of mother to child transmission of HIV in Nigeria: a synthesis of the literature. BMC Pub Health 2014; 14: 771.

28. Okoli JC, Lansdawn GE. Barriers to successful implementation of prevention of mother to child transmission (PMTCT) of HIV programmes in Malawi and Nigeria: a critical literature review study. Pan Afr Med J 2014;

19:154 doi:10.11604/pamj.2014.19.154.4225. 